\title{
Proton induced reactions in search for kaonic nuclear clusters
}

\author{
K. Suzuki ${ }^{a}$, L. Fabbietti ${ }^{a}$, P. Kienle ${ }^{a, b}$, N. Herrmann ${ }^{c}$, \\ T. Yamazaki ${ }^{d}$ and J. Zmeskal ${ }^{b}$ \\ ${ }^{a}$ Physik Department, TU München, Germany

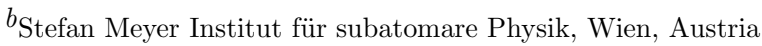 \\ ${ }^{c}$ Physikalisches Institut der Universität Heidelber, Germany \\ $d_{\text {Heavy-Ion Nuclear Physics Laboratory, RIKEN, Japan }}$
}

\begin{abstract}
Recently, exotic nuclear systems involving a $\bar{K}\left(K^{-}\right.$or $\left.\bar{K}^{0}\right)$ as a constituent (such as $p p K^{-}, p p n K^{-}, p p p K^{-}, p p p n K^{-}$and $p p n n K^{-}$) were predicted by Akaishi and Yamazaki to have narrow discrete bound states with large binding energies as much as $100 \mathrm{MeV}$ due to the strong $K^{-}-p$ attraction. We are going to perform an experiment to identify such $\bar{K}$ systems with proton induced reactions by making use of the FOPI detector at GSI in identifying both $K^{0}$ mesons from the invariant mass of $\pi^{+}+\pi^{-}$and $\Lambda$ hyperons from the invariant mass of $p+\pi^{-}$. We use $3.5-4.5 \mathrm{GeV}$ protons on a deuterium target for $p p K^{-}$where the key elementary reaction is $p+n \rightarrow \Lambda(1405)+K^{0}+p$. A missing-mass spectrum is constructed for $p p K^{-}$in the reaction: $p+d \rightarrow$ $[\Lambda(1405) p]+K^{0}+p \rightarrow p p K^{-}+K^{0}+p$, and simultaneously an invariant mass spectrum for $p p K^{-} \rightarrow \Lambda+p$ can be obtained.
\end{abstract}

\section{Introduction}

Recently, exotic nuclear systems involving a $\bar{K}\left(K^{-}\right.$. or $\left.K^{0}\right)$ as a constituent have been investigated theoretically $[1,2,3,4,5]$ based on phenomenologically constructed $\bar{K} N$ interactions, which reproduce low energy $\bar{K} N$ scattering data [6], kaonic hydrogen atom data [7] and the binding energy and decay width of $\Lambda(1405)$ which is regarded as a bound system of $p+K^{-}$. These interactions, which are consistent with the prediction based on a chiral SU(3) effective Lagrangian [8] as well as the recent experimental indication on decreased in-medium $K^{-}$mass from subthreshold nuclear reactions $[9,10]$, are characterized by a strongly attractive $\mathrm{I}=0$ part, causing drastic shrinkage of $\bar{K}$-bound nuclei 
and increasing the binding energies in proton-rich nuclei. The predicted bound states in ppn $K^{-}$, ppnn $K^{-}$and ${ }^{8} \mathrm{Be} K^{-}$lie below the emission threshold of $\Sigma+\pi$ and thus are expected to have narrow decay widths. These few-body treatments have been further extended to more complex systems by the method of Antisymmetrized Molecular Dynamics (AMD) [4], which is now capable of calculating the structure with density distributions of individual constituents $\left(\mathrm{p}, \mathrm{n}, K^{-}\right)$in an ab initio way without a priori assumption on the structure. The predicted $\bar{K}$ bound states have enormous average nucleon densities, 3 times the normal nuclear density, with large binding energies. Very recently, double $K^{-}$systems, pp $K^{-} K^{-}$and $\operatorname{ppn} K^{-} K^{-}$, have also been predicted to be high-density systems [3]. Such compact nuclear systems, which can be called "Kaonic nuclear clusters", may be beyond the scope of the present theoretical treatment based on the hadronic structure and interactions, as they are likely to be in a new bound low-temperature phase of quarks and gluons (so called color superconductivity phase).

Experimental methods to produce kaonic nuclei by direct reactions, such as (stopped $K^{-}$, n) [11], $\left(K^{-}, \mathrm{n}\right)[12]$ and $\left(K^{-}, \pi^{-}\right)$and $\left(\pi^{+}, K^{+}\right)[2]$ are proposed, and some trials are in progress. Recently, an experimental indication was obtained on the predicted $p p n K^{-}$ from the ${ }^{4} \mathrm{He}$ (stopped- $K^{-}, \mathrm{n}$ ) experiment at KEK [13]. The neutron energy distribution showed a mono-energetic peak, which can be interpreted as a $\mathrm{T}=0 \mathrm{ppn} K^{-}$system. More recently, a deeper and narrow bound state has been discovered on $p n n K^{-}$from ${ }^{4} \mathrm{He}$ (stopped- $K^{-}, \mathrm{p}$ ), which is an evidence for the existence of charge 0 , strangeness -1 , baryon number 3 system with isospin 1 . The mass and the width are determined to be $3117.0_{-4.4}^{+1.5} \mathrm{MeV} / \mathrm{c}^{2}$ and $<21 \mathrm{MeV} / \mathrm{c}^{2}$, which was named $\mathrm{S}^{0}(3115)$ [14]. However the observed $\bar{K}$ binding energies are about $100 \mathrm{MeV}$ larger than the predicted ones. These two states are interpreted within the framework of deeply bound $\bar{K}$ on shrunk nuclei by taking into account the relativistic effect on $\bar{K}$ and additionally by incorporating an enhanced bare $\bar{K} \mathrm{~N}$ interaction by $15 \%$ in the dense nuclear medium [15], as shown in Fig. 1. A large spin-orbit splitting $\left(0 p_{3 / 2}-0 p_{1 / 2}\right)$ as much as $60 \mathrm{MeV}$ is also predicted in this dense system. In view of these new findings it is vitally important to explore new experimental methods to obtain more systematic experimental data on $\bar{K}$ clusters. We hire proton induced reaction to produce and identify $\bar{K}$ clusters by making use of the FOPI detector at GSI by identifying both $K^{0}$ mesons from the invariant mass of $\pi^{+}, \pi^{-}$and hyperons from the invariant mass of $\mathrm{p}+\pi^{-}$. Detection of a $K^{0}$ implies that and $\mathrm{S}=-1$ object (including a free hyperon) is produced, and detection of a $\Lambda$ is related to a decay of some $\mathrm{S}=-1$ object. Nearly $4 \pi$ coverage of acceptance of FOPI detector and a capability of tracking all the charged particles will allow us to reconstruct missing-mass (MM) spectra and invariant-mass (IM) spectra from the decay particles of the $\bar{K}$ clusters.

\section{Experimental procedures}

\subsection{5-4.5 GeV protons on a deuterium target for $p p K^{-}$}

We first start with deuteron target with a proton beam of $3.5-4.5 \mathrm{GeV}$ to produce the most basic $\bar{K}$ cluster, $p p K^{-}$. The key elementary reactions are

$$
p+{ }^{\prime \prime} n^{\prime \prime} \rightarrow \Lambda(1405)+K^{0}+p,
$$




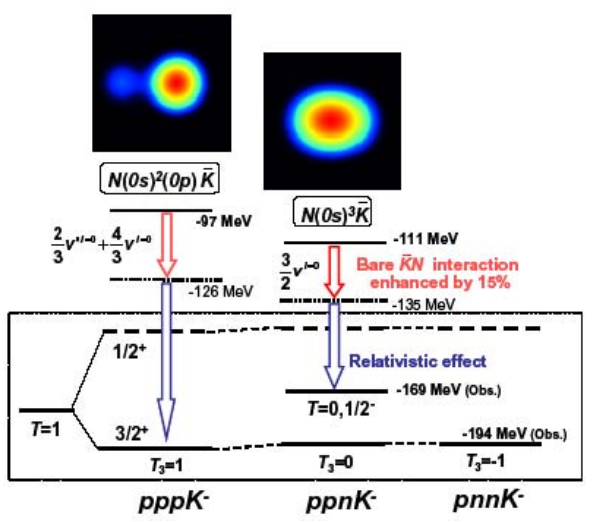

Figure 1: Spin-isospin structure of the strange tribaryon system $[(N N N) \bar{K}]_{\left(T, T_{3}\right)}^{Q}$. The previously calculated nucleon-density contours and energy levels with $\mathrm{E}_{K}$ values are shown on top. The observed $S^{0}(3115)$ and $S^{1}(3140)$ are identified as the $\left(\mathrm{T}, \mathrm{T}_{3}\right)=(1,-1)$ and $\mathrm{T}=0$ states, respectively. The experimental energies are accounted for by the relativistic effect and by invoking medium-modified bare $\bar{K} \mathrm{~N}$ interactions with an enhancement of 1.15. A large spin-orbit splitting as much as $60 \mathrm{MeV}$ is expected for each $\mathrm{T}=1$ triplet. From Akaishi et al. [15].

$$
p+{ }^{\prime \prime} p^{\prime \prime} \rightarrow \Lambda(1405)+K^{+}+p,
$$

where " $n$ " and " $p$ " are a neutron and a proton in a target nucleus and $\Lambda(1405)=p K^{-}=$ $n \bar{K}^{0}$. The elementary cross sections, though not well known, can be estimated to be 20 $\mu \mathrm{b}$ by using an experimental spectrum of $\Lambda, \Sigma, \Sigma(1385)+\Lambda(1405)$ at $3.5 \mathrm{GeV} / \mathrm{c}$ taken by DISTO at Saclay [16], combined with the $N N$ cross section for $\Lambda K^{+} N$ (around $200 \mu \mathrm{b}$ ). In the reaction with deuteron, $p+\Lambda(1405)$ serves as a doorway to form $p p K^{-}$as follows,

$$
p+d \rightarrow[p+\Lambda(1405)]+K^{0}+p_{s} \rightarrow p p K^{-}+K^{0}+p_{s}
$$

where $p_{s}$ denotes a spectator proton which comes from the target $d$. In this reaction a missing-mass spectrum for $p p K^{-}$can be constructed from the energies and momenta of the incident $p$ and the emitted $K^{0} \rightarrow \pi^{+}+\pi^{-}$and $p_{s}$. The cross section for this process is being studied theoretically [17]. As a naive estimate we adopt a coalescence model, in which $p$ and $\Lambda(1405)$ with a relative momentum less than $300 \mathrm{MeV} / \mathrm{c}$ form a $p p K^{-}$. This probability is about $1 \%$ at an incident energy of $4.5 \mathrm{GeV}$, and increases with the decrease of the incident energy, due to the lowering of the $\Lambda(1405)$ momentum distribution as shown in Fig. 2. We expect an incident energy of $3.5 \mathrm{GeV}$ may be an optimum one, considering the formation probability $(2 \%)$ and the $\Lambda(1405)$ production cross section. The estimated formation cross section for $p p K^{-}$at $3.5 \mathrm{GeV}$ is around $0.4 \mu \mathrm{b}$. Once the $p p K^{-}$ is identified in the formation channel (MM spectrum), its decay pattern, such as

$$
p p K^{-} \rightarrow \Lambda+p
$$



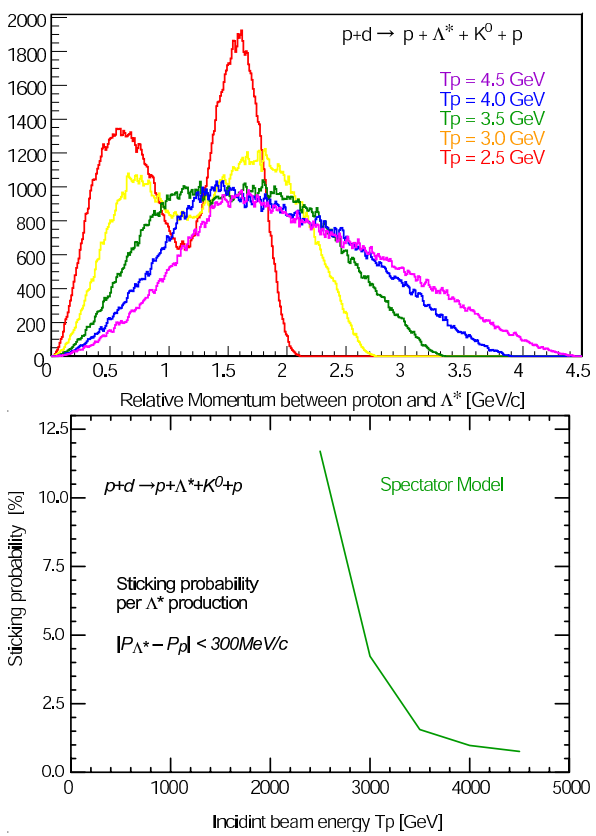

Figure 2: (Upper) Relative momentum between the proton and $\Lambda(1405)$ momentum in a $\mathrm{p}+\mathrm{d}$ reaction at proton incident energies of $2.5,3.0,3.5,4.0$ and $4.5 \mathrm{GeV}$. (Lower) Sticking probability for $\mathrm{p}+\Lambda(1405)$.

can be studied. Simultaneously, an IM spectrum for the decaying $p p K^{-}$can be reconstructed. We can therefore determine the decay branching ratios and decay particle correlations, which are pertinent to the internal structure of the condensed system.

\subsection{5-4.5-GeV protons on ${ }^{12} \mathrm{C}$ target}

Next, we use cascade reactions of a proton beam on a nuclear target to produce and identify other $\bar{K}$ clusters.

The $\Lambda(1405)$ produced in the initial reaction (Eq. 1,2$)$ at $T_{p}=3.5-4.5 \mathrm{GeV}$ has a broad momentum distribution which is peaked around $1000 \mathrm{MeV} / \mathrm{c}$ (depending on the incident energy, as shown in the upper panel of Fig. 2), and will collide with a nucleon in the residual nucleus successively until the kinetic energy reaches some $100 \mathrm{MeV}$, where successive knock-on reactions, such as

$$
\begin{gathered}
\Lambda(1405)+" p N^{\prime \prime} \rightarrow p p K^{-}+N, \\
\Lambda(1405)+" p n N^{\prime \prime} \rightarrow p p n K^{-}+N, \\
\Lambda(1405)+" p p N^{\prime \prime} \rightarrow p p p K^{-}+N,
\end{gathered}
$$


take place.

The momentum transfer to form a $\bar{K}$ nucleus in this knock-on process is rather low (up to $300 \mathrm{MeV} / \mathrm{c}$ ) as shown in the lower panel of Fig. 2. Therefore, $\Lambda(1405)$ can be soft landed, and the yield of producing $\bar{K}$ nuclei is rather high. This process is being studied theoretically [17], and for the time being, it is assumed to be $1 \%$ of the elementary process, namely, $0.2 \mu \mathrm{b}$.

Other processes, based on a " $\bar{K}$-transfer" process, may also take place [17]:

$$
\begin{gathered}
\left(p K^{-}\right)+{ }^{\prime \prime} p p^{\prime \prime} \rightarrow p p K^{-}+p, \\
\left(p K^{-}\right)+^{\prime \prime} p p n^{\prime \prime} \rightarrow p p n K^{-}+p, \\
\left(p K^{-}\right)+^{\prime \prime} p p p^{\prime \prime} \rightarrow p p p K^{-}+p, \\
\left(n \bar{K}^{0}\right)+^{\prime \prime} p n^{\prime \prime} \rightarrow p n \bar{K}^{0}+n, \\
\left(n \bar{K}^{0}\right)+^{\prime \prime} p n n^{\prime \prime} \rightarrow p n n \bar{K}^{0}+n, \\
\left(n \bar{K}^{0}\right)+{ }^{\prime \prime} p p n^{\prime \prime} \rightarrow p p n \bar{K}^{0}+n .
\end{gathered}
$$

In this process, the $\bar{K}$ in $\Lambda(1405)(=p \bar{K})$ is stripped with a small transferred momentum, about $1 / 3$ of the momentum of $\Lambda(1405)$. Since the momentum transfer of $\bar{K}$ is so small, $\bar{K}$ clusters are expected to be formed abundantly. For example, the cross section for the process (Eq. 8) as a function of the $\Lambda(1405)$ momentum, where a fictitious $\Lambda(1405)$ beam hits a fictitious target " $p p$ ", is shown in the middle panel of Fig. 3. The optimum incident energy may be around $3.5 \mathrm{GeV}$.

Invariant-mass spectroscopy can be carried out for the decay processes (Eq. 4), (Eq. 14) and (Eq. 15).

$$
\begin{gathered}
p p n K^{-}(T=0) \rightarrow \Lambda+d, \\
p p p K^{-}(T=1) \rightarrow \Lambda+p+p .
\end{gathered}
$$

\section{Experimental set up}

The FOPI detector system [18] at the SIS accelerator facility of GSI (Darmstadt) is a general purpose detector complex for fixed target experiments. It has a nearly complete azimuthal symmetry and almost full geometrical acceptance $(4 \pi)$. The FOPI system is capable to detect simultaneously charged reaction products, heavy fragments of $\mathrm{Z}$ up to 20, light fragments (protons, deuterons, tritons) and mesons (pions, kaons, etc). Particles are identified and their four momenta are determined by the measurement of the specific energy loss in the detector material $(d E / d x)$, the track curvature radius in the magnetic field, and the velocity derived from the information on time-of-flight from the target position to the detector. Short-lived neutral particles $\left(\Lambda, K^{0}\right)$ are identified by invariant mass reconstruction of their decay products [19]. The FOPI detector system consists of four sets of sub-detectors covering certain polar angles: From $30^{\circ}<\theta<150^{\circ}$ a central drift chamber (CDC) for $d E / d x$ and $\rho$ measurement and a cylindrical plastic scintillator barrel (Barrel) for TOF determination, a forward drift chamber (Helitron) $5^{\circ}<\theta<30^{\circ}$ 

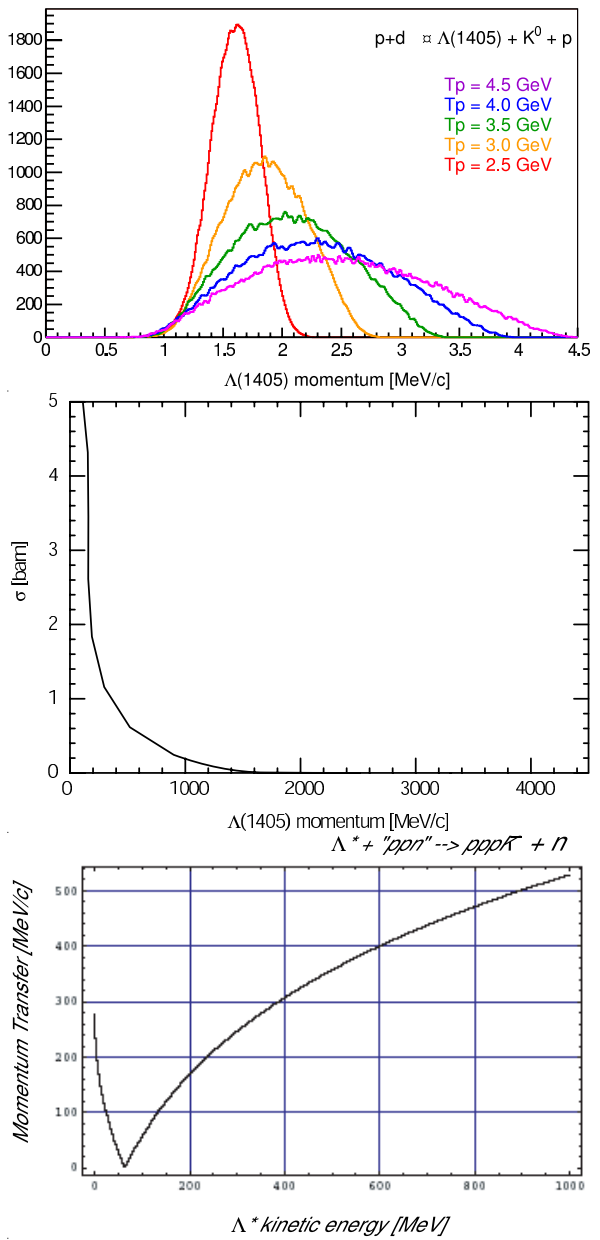

Figure 3: (Upper) Momentum distributions of $\Lambda(1405)$ at various incident proton energies. (Middle) Anti K-transfer cross section in the cascade reaction $\Lambda(1405)+$ " $p p^{\prime \prime} \rightarrow$ $p p K^{-}+p$ as a function of the $\Lambda(1405)$ momentum. (Lower) Momentum transfer in the cascade reaction as a function of the $\Lambda(1405)$ kinetic energy. 
for $d E / d x$ and $\rho$ measurement, a forward plastic wall (PLAWA) covering an angular range of $1.25^{\circ}<\theta<5^{\circ}$ and a zero degree plastic scintillator array (ZD).

For the $p+d$ reaction to study $p p K^{-}$we are going to install a liquid Deuterium target system. From a geometrical constraint, the target cell must be inserted from the upstream side of the beam line with a rather long liquid Deuterium transfer line to the target chamber. The target cell has a dimension of $10 \mathrm{~mm}$ diameter and $40 \mathrm{~mm}$ length with a cylindrical shape. It is specially designed using thin kapton foils $(50 \mu \mathrm{m})$. Hence the material thickness of the target is kept very low to minimize energy loss deterioration. At the top of the target cell cooling lines are connected to feed liquid $\mathrm{D}_{2}$ in and let the gaseous $\mathrm{D}_{2}$ out of the target cell. The charged particle multiplicity ( $\mathrm{cmul}$ ) distribution

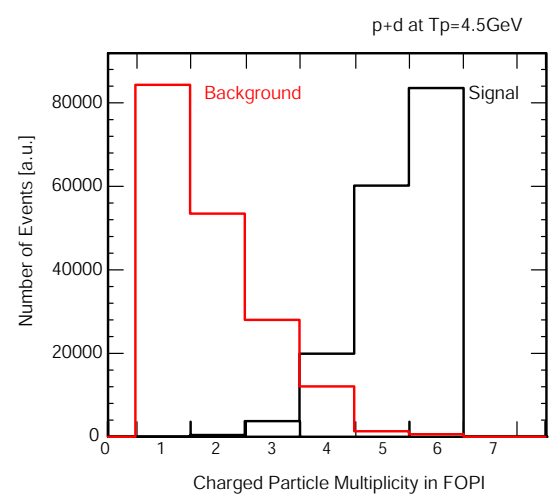

Figure 4: Charged particle multiplicity distribution for the signal and the background from $p+d$ collisions. The number of charged particles per event has been obtained counting all the particles that are emitted within the FOPI geometrical acceptance.

for the background and the signal is shown in Fig. 4, where all the charged particles falling in the FOPI acceptance are counted. One can see that the background events are characterized by a $\mathrm{cmul} \leq 4$, on the contrary the signal channel is characterized by a $c m u l \geq 5$. Setting the online trigger so as to accept only events with cmul $\geq 5$ in the acceptance the background events are reduced by a factor 8000 and the quasi free events by a factor 1000 compared to the events that contain the $p p K^{-}$signal.

\section{Conclusion}

An interest on the recently predicted so-called "Kaonic nuclear cluster" is certainly growing these days. Intensive works in both theoretical and experimental side are on going. We are going to perform a series of measurements by a proton induced reaction with FOPI detector system at GSI, $p p K^{-}$with deuterium target and tribaryon system or more heavier system with a carbon target. First beamtime in search for the $p p K^{-}$system is 
planned in late this year. Invariant mass technique with a superior tracking capability of FOPI in addition to missing mass technique is a big advantage.

\section{References}

[1] Y. Akaishi and T. Yamazaki, Phys. Rev. C 65 (2002) 044005.

[2] T. Yamazaki and Y. Akaishi, Phys. Lett. B 535 (2002) 70.

[3] T. Yamazaki, A. Doté and Y. Akaishi, Phys. Lett. B587 (2004) 167.

[4] A. Doté, H. Horiuchi, Y. Akaishi and T. Yamazaki, Phys. Lett. B590 (2004) 51.

[5] A. Doté, H. Horiuchi, Y. Akaishi and T. Yamazaki, Phys. Rev. C 70 (2004) 044313.

[6] A. D. Martin, Nucl. Phys. B179 (1981) 33.

[7] M. Iwasaki et al., Phys. Rev. Lett. 78 (1997) 3067; T. M. Ito et al., Phys. Rev. C 58 (1998) 2366.

[8] T. Waas, N. Kaiser, and W. Weise, Phys. Lett. B 365, 12 (1996); Phys. Lett. B 379, 34 (1996); N. Kaiser, P. B. Siegel, and W. Weise, Nucl. Phys. A594, 325 (1995); W. Weise Nucl. Phys. A610, 35 (1996).

[9] A. Schröter et al., Z. Phys. A 350 (1994) 101.

[10] F. Laue et al., Phys. Rev. Lett. 82 (1999) 1640; Euro. Phys. J. A 9 (2000) 397.

[11] M. Iwasaki et al., Nucl. Instr. Methods A 473 (2001) 286.

[12] T. Kishimoto, Phys. Rev. Lett. 83(1999) 4701.

[13] M. Iwasaki et al.. submitted to Phys. Lett. B (ArXiv-nucl-ex/0310018).

[14] T. Suzuki et al., Phys. Lett. B 597 (2004) 263.

[15] Y. Akaishi, A. Dote and T. Yamazaki, submitted to Phys. Lett. B (ArXiv:nuclth/0501040).

[16] M. Maggiora et al., Nucl. Phys. A691 (2001) 329c.

[17] Y. Akaishi, private communication (2005).

[18] J.L. Ritman et al., FOPI Collaboration, Nucl. Phys. Proc. Suppl. 44 (1995) 708.

[19] FOPI collaboration; R. Kutsche, Ph. D. Thesis. 